\title{
Maternal genetic contribution to pre-pregnancy obesity, gestational weight gain, and gestational diabetes mellitus
}

\author{
Selvihan Beysel ${ }^{1,2,6^{*}} \mathbb{0}$, Nilnur Eyerci ${ }^{3}$, Mustafa Ulubay $^{4}$, Mustafa Caliskan ${ }^{1}$, Muhammed Kizilgul ${ }^{1}$, \\ Merve Hafızoğlu ${ }^{5}$ and Erman Cakal ${ }^{1}$
}

\begin{abstract}
Introduction: Pre-pregnancy obesity, gestational diabetes mellitus (GDM), and gestational weight gain (GWG) are associated with each other. This is the first study to investigate whether genetic variants were associated with having GDM, and whether genetic variants-related GDM were associated with adiposity including pre-pregnancy obesity and excessive GWG in Turkish women.

Patients and methods: Women with GDM $(n=160)$ and without GDM $(n=145)$ were included in case-controlled study. Genotyping of the HNF1A gene (p.I27L rs1 169288, p.98V rs1800574, p.S487N rs2464196), the VDR gene (p.Bsml rs1544410, p.Apal rs7975232, p.Taql rs731236, p.Fokl rs2228570), and FTO gene (rs9939609) SNPs were performed by using RT-PCR.

Results: The FTO AA genotype was associated with an increased risk of having GDM (AA vs. AT + TT, 24.4\% vs. 12.4\%, $\mathrm{OR}=2.27,95 \% \mathrm{Cl}[1.23-4.19], \mathrm{p}=0.007)$. The HNF1A p.I27L GT/TT genotype was associated with increased GDM risk (GT+TT vs. GG-wild, 79.4\% vs. 65.5\%, OR=2.02,95\% Cl 1.21-3.38], $\mathrm{p}=0.007$ ). However, all VDR gene SNPs and the HNF1A p.A98V, p.S487N were not associated with having GDM ( $p>0.05)$. The FTO AA genotype was associated with an increased risk for pre-pregnancy overweight/obesity $(\mathrm{OR}=1.43,95 \% \mathrm{Cl}[1.25-3.4], \mathrm{p}=0.035)$, but not associated with excessive GWG after adjusting for pre-pregnancy weight ( $p>0.05$ ). Pre-pregnancy weight, weight at delivery, and GWG did not differ in both VDR and HNF1A gene carriers ( $p>0.05$ ). HOMA-IR and HbA1c were increased in both p.127L TT and FTO AA genotype carriers $(p<0.05)$.

Conclusion: The adiposity-related gene FTO is associated with GDM by the effect of FTO on pre-pregnancy obesity. The diabetes-related p.I27L gene is associated with GDM by increasing insulin resistance.
\end{abstract}

Keywords: Gestational weight gain, Polymorphisms, Gestational diabetes, Pre-pregnancy obesity

\section{Introduction}

Maternal obesity and gestational diabetes mellitus (GDM) is a growing public health problem worldwide [1]. The Institute of Medicine (IOM) developed guidelines for gestational weight gain (GWG) during pregnancy; however, no specific recommendations could be made for GDM and multiethnic differences [2, 3]. Both

\footnotetext{
*Correspondence: beyselselvihan@gmail.com; sbeysel@aku.edu.tr ${ }^{6}$ Department of Endocrinology and Metabolism, Afyonkarahisar Saglik Bilimleri University, Afyon, Turkey

Full list of author information is available at the end of the article
}

pre-pregnancy obesity and excessive GWG are related to increased risk of maternal obesity and GDM [3]. Becoming pregnant or gaining too much weight during pregnancy are the risk factors for adverse perinatal complications and increased risk for future metabolic disease in overweight/obese women, both for the mothers and their offspring [1, 4]. Pre-pregnancy obesity and excessive GWG may have additive negative impact on maternal and neonatal outcomes in women with GDM $[5,6]$. Pre-pregnancy obesity, gestational diabetes, and excessive GWG are associated with multiple factors such as the environment, behavior, and genetics; however, 
understanding these associations is complex [1,3]. Diabetes-related or maternal and/or fetal adiposity-related genetic variants have been associated with GDM, prepregnancy weight, and GWG during pregnancy [7-9]. Kawai et al. reported that common type 2 diabetes risk variants were associated with increased risk of GDM [8]. Genetic variants were associated with GDM and progression to pre-diabetes and type 2 diabetes mellitus in women with prior GDM [9]. Evidence has been presented for a genetic predisposition to GDM risk and also a change in GWG during pregnancy [7, 10-13], and gene-environment interactions could explain the variation in GWG and GDM.

The fat mass and obesity-associated gene (FTO) rs9939609 single nucleotide polymorphism (SNP) was associated with increased risk of obesity and type 2 diabetes, as well as GDM [10]. The FTO SNPs have been reported to be associated with pre-pregnancy obesity [8] and excessive GWG [11]. The FTO variants related to type 2 diabetes are mediated by the effect of the FTO gene on body mass index (BMI); however, the exact mechanisms of this relation have not been identified [10, 11]. Vitamin $D$ shows its cellular activity by binding to vitamin D receptors (VDR). VDR, as a transcription factor, has a role in the regulation of insulin secretion from pancreatic beta cells [14]. VDR has effect on proliferation, differentiation, and activation of immune cells and cytokine production, and subsequently type 2 diabetes occurs $[15,16]$. Hepatocyte nuclear factor 1A (HNF1A), as a transcription factor, has a role in the function of pancreas beta cells [17]. Endocrine and exocrine pancreatic cells express HNF1A in the developmental stage. HNF1A is necessary for the glucose response to insulin secretion and glucose metabolism [18]. Women with HNF1A mutation are diagnosed as having monogenic form of diabetes type 3 (MODY3), and these women usually present with GDM, and diabetes persisting after delivery [17-19].

This is the first study to investigate the effect of $H N F 1 A$ gene, $V D R$ gene, and FTO gene variants on having GDM, pre-pregnancy obesity, and excessive GWG in Turkey. We aimed to examine whether these genetic variants would associate with having GDM, and then, whether the genetic variants that associated with GDM would associate with adiposity including pre-pregnancy obesity and excessive GWG. The $V D R$ gene (encoding as SNPs p.BsmI, p.ApaI, p.TaqI, and p.FokI), and, HNF1A gene (encoding as SNPs p.I27L, p.A98V, and p.S487N) were chosen because these genetic variants have been reported to be associated with type 2 diabetes, as well as GDM risk [12, 14-20]. We also investigated the obesityrelated FTO gene rs9939609 SNP because it is associated with both GDM and gestational body weight during pregnancy $[10,13,20]$. Genetic variants are implicated in the pathogenesis of GDM. Evidence suggests the genetic alterations in genes responsible for metabolic changes during pregnancy predispose to GDM [7]. We also hypothesized that these diabetes and adiposity-related genetic variants would likely be associated with GDM risk and gestational body weight during pregnancy.

\section{Patients and methods \\ Study population}

Pregnant women referred to tertiary hospital, Obstetrics and Gynecology Clinic, Ankara, from 2015 to 2016, were included in this case-control study. Women with GDM $(n=160)$ and age- and gestational age-matched women without GDM as controls $(n=145)$ were included in the study. Gestational age was assessed from the date of the last menstrual period and clinical assessment. A 2-h, 75 -g oral glucose tolerance test at 24 to 28 weeks gestation age was performed for all pregnant women, irrespective of family history of DM or any other risk factors for GDM. Glucose concentrations after fasting, and 1 and $2 \mathrm{~h}$ after glucose administration $<92 \mathrm{mg} / \mathrm{dl},<180 \mathrm{mg} /$ $\mathrm{dl}$, and $<153 \mathrm{mg} / \mathrm{dl}$, respectively, were considered normal. When the pregnant women's glucose concentration was higher than any of these values, the women were diagnosed as having GDM [12]. Women whose GDM was diagnosed according to these criteria, aged 22-38 years, and whose pregnancy age was $24-48$ weeks were included in the study. Women with GDM who had pre-existing type 2 diabetes, GDM observed in prior pregnancy, GDM with chronic disease such as hypertension, thyroid disorders, cardiac, hepatic or renal dysfunction were excluded. Women aged $22-38$ years and with pregnancy age 24-28 weeks, with no GDM, type 2 diabetes, hypertension, thyroid disorders, cardiac, hepatic or renal dysfunction were accepted as controls and included in the study. Treatment of diet with or without insulin therapy was recorded. Weight, height, and systolic (SBP) and diastolic blood pressure (DBP) were measured in all participants. Body mass index $\left(B M I, \mathrm{~kg} / \mathrm{m}^{2}\right)$ was calculated as weight $(\mathrm{kg}) / \mathrm{height}^{2}\left(\mathrm{~m}^{2}\right)$. Women were categorized as underweight $\left(\mathrm{BMI}<18.5 \mathrm{~kg} / \mathrm{m}^{2}\right)$, normal weight $\left(\mathrm{BMI}=18.5-24.9 \mathrm{~kg} / \mathrm{m}^{2}\right)$, overweight $(\mathrm{BMI}=25-29.9 \mathrm{~kg} /$ $\mathrm{m}^{2}$ ), and obese $\left(B M I \geq 30 \mathrm{~kg} / \mathrm{m}^{2}\right)$. Maternal weight before pregnancy, pre-pregnancy weight, was obtained through a questionnaire. Maternal weight was measured at delivery. Gestational weight gain (GWG) was calculated as the difference between the maternal weight at delivery and pre-pregnancy weight. The recommended GWG was calculated based on IOM guidelines related with prepregnancy BMI: underweight, a gain of $12.5-18 \mathrm{~kg}$; normal weight, a gain of $11.5-16 \mathrm{~kg}$; overweight, a gain of 7-11.5 kg; and obese, a gain of 5-9 kg. After this, GWG was divided into three categories: low, if the weight was 
below the recommendation; adequate, if the weight gain was within the recommendation; and high, if the weight gain was above the recommendation [21]. Serum glucose, insulin, and glycated hemoglobin $\left(\mathrm{HbA}_{1 \mathrm{c}}\right)$ concentrations were measured at 24-28 weeks of pregnancy. Insulin resistance was calculated using the homeostasis model assessment-insulin resistance (HOMA-IR): [fasting plasma insulin $(\mu \mathrm{IU} / \mathrm{ml}) \times$ fasting plasma glucose $(\mathrm{mg} /$ dl)]/405 [12]. This study was approval by Diskapi Yildirim Beyazit Teaching and Training Research Hospital Ethics Board (Number. 24.04.2015-13/25). Written informed consent was obtained from each participant.

\section{Genotyping}

Genetic analyses for the VDR gene SNPs p.FokI (rs2228570), p.BsmI (rs1544410), p.ApaI (rs7975232), and p.TaqI (rs731236) and the HNF1A gene SNPs p.S487N (rs2464196, p.Ser486Asn), p.A98V (rs1800574, p.Ala98Val), p.I27L (rs1169288, p.Ile27Leu) and the FTO gene rs939609 SNPs were performed using real-time polymerase chain reaction (RT-PCR) amplification. Genomic DNA was isolated from collected peripheral blood samples of the subjects using DNA Isolation Kit (Roche Diagnostics, Indianapolis, IN, USA). Genotyping of each SNP in the $V D R$ gene, HNF1A gene, and FTO gene was independently conducted using a pre-validated fluorescence-based allele-specific PCR assay, KASPar (KBiosciences, Hoddesdon, UK) and performed on a Rotor-Gene Q real-time cycler (Qiagen, Hilden, Germany) according to the manufacturer's instruction. Allele discrimination was made using Rotor-Gene $\mathrm{Q}$ software v.2.3.1 (Qiagen, Hilden, Germany). The genotype calling was performed blind without information on the clinical phenotypes.

\section{Statistical analysis}

Statistical analysis was performed using the SPSS 18.0 (SPSS, Inc) software. Variables are presented as mean \pm standard deviation (SD) or median (min-max), percentages (\%), odds ratios (OR), 95\% confidence intervals $(\mathrm{CI})$. Normality was tested using the KolmogorovSmirnov and Shapiro-Wilk $W$ test. SNPs are expressed as allelic frequency (q) or prevalence of genotypes (\%). Categorical variables were analyzed using the Chi-square test or Fisher's exact test, where appropriate. Student's $t$-test was used for normally distributed continuous variables or log-transformed variables between two groups. The Hardy-Weinberg equilibrium (HWE) at individual loci was assessed using the Chi-square test. Multiple logistic regression analysis and the Chi-square test or Fisher's exact test was tested using models and ORs were calculated: dominant (major allele homozygotes vs. heterozygotes + minor allele homozygotes), recessive (major allele homozygotes + heterozygotes vs. minor allele homozygotes) and codominant (major allele homozygotes vs. heterozygote and minor allele homozygotes vs. major allele homozygotes). Pair-wise linkage disequilibrium (LD) and correlation coefficients $\left(\mathrm{r}^{2}\right)$ were analyzed using the HAPLOVIEW program. We made a variable reflecting all possible combinations of genotypes for each SNP. Power analysis was performed using web-based software http://osse.bii.a-star.edu.sg/calculation2.php. The power of study was $65 \%$. Statistical significance was defined as $p<0.05$.

\section{Results}

The mean age, gestational age, and height were similar between the women with GDM and controls ( $p>0.05$ ). Pre-pregnancy overweight/obesity were increased in women with GDM compared with controls $(\mathrm{p}<0.05)$. Weight at delivery and excessive GWG were increased in women with GDM compared with the controls $(\mathrm{p}<0.05)$. Serum glucose, insulin, HOMA-IR, and HbA1c were increased in women with GDM compared with the controls $(\mathrm{p}<0.05$, each). The clinical features of the subjects are shown in Table 1 . Minor allele frequency of the HNF1A, VDR, and FTO genes is shown in Table 2. These frequencies were in HWE except p.A98V. Haploview analysis showed that the HNF1A, VDR, and FTO genes were not in LD. The risk alleles of the HNF1A gene (p.S487N, and p.A98V) and, $V D R$ gene (p.ApaI, p.TaqI, p.BsmI and p.FokI) were similar between women with GDM and the controls ( $>>0.05$, each). Genotype analysis is shown in Table 3.

The FTO gene rs9939609 distribution was TT-wild, heterozygote AT, and homozygote AA at $50.3 \%, 37.2 \%$, and $12.4 \%$ in the controls, and $36.9 \%, 38.8 \%$, and $24.4 \%$ in women with GDM $(\mathrm{p}=0.011)$. The FTO gene AA genotype was associated with an increased risk of GDM more than the TT/AT genotype in co-dominant, dominant, and recessive models (dominant: $\mathrm{AT}+\mathrm{AA}$ vs. TT-wild, $63.1 \%$ vs. $49.7 \%$, OR $=1.73,95 \%$ CI $[1.12-$ 2.74], $\mathrm{p}=0.018$, and recessive: AA vs. $\mathrm{AT}+\mathrm{TT}, 24.4$ vs. $12.4 \%, \mathrm{OR}=2.27,95 \%$ CI $[1.23-4.19], \mathrm{p}=0.007$ ) (Table 3). The FTO AA/AT genotype had a greater association with pre-pregnancy overweight/obesity than TT-wild genotype $(\mathrm{p}<0.05)$ (Table 4). Pre-pregnancy weight $(\mathrm{p}<0.05)$ and weight at delivery $(\mathrm{p}<0.05)$ progressively increased from the AA genotype to the TT genotype. GWG was increased in AT/AA genotype compared with the TT genotype $(\mathrm{p}<0.05)$. Serum glucose, insulin, HOMA-IR, and HbA1c were higher in the AA genotype compared with the TT genotype $(\mathrm{p}<0.05)$. The FTO AA genotype was associated with a greater risk of pre-pregnancy overweight/obesity compared with AT/TT genotypes (OR $=1.43,95 \%$ CI $[1.25-3.4], \mathrm{p}=0.035)$. The $F T O$ AA genotype was 
Table 1 Characteristics of subjects

\begin{tabular}{|c|c|c|c|}
\hline & Controls $(n=145)$ & $\begin{array}{l}\text { Gestational diabetes mellitus } \\
(n=160)\end{array}$ & $\mathrm{p}$ \\
\hline Age (year) & $28.25 \pm 5.15$ & $29.35 \pm 5.36$ & 0.075 \\
\hline Gestational age (weeks) & $26.27 \pm 1.48$ & $25.99 \pm 1.65$ & 0.137 \\
\hline Height (cm) & $160.40 \pm 5.71$ & $159.21 \pm 5.95$ & 0.076 \\
\hline Pre-pregnancy weight (kg) & $61.74 \pm 11.98$ & $76.21 \pm 11.27$ & 0.001 \\
\hline Pre-pregnancy BMI $\left(\mathrm{kg} / \mathrm{m}^{2}\right)$ & $24.06 \pm 4.82$ & $30.21 \pm 5.10$ & 0.001 \\
\hline Pre-pregnancy BMI (\%) & & & 0.001 \\
\hline Underweight $\left(<20 \mathrm{~kg} / \mathrm{m}^{2}\right)$ & 23.4 & 3.8 & \\
\hline Normal weight $\left(20-24.9 \mathrm{~kg} / \mathrm{m}^{2}\right)$ & 38.6 & 8.8 & \\
\hline Overweight (25-29.9 kg/m²) & 26.2 & 34.4 & \\
\hline Obesity $\left(\geq 30 \mathrm{~kg} / \mathrm{m}^{2}\right)$ & 11.7 & 53.1 & \\
\hline Pre-pregnancy overweight/obesity (\%) & 37.9 & 87.5 & 0.001 \\
\hline Weight at delivery (kg) & $77.60 \pm 12.59$ & $87.58 \pm 11.54$ & 0.001 \\
\hline BMI at delivery $\left(\mathrm{kg} / \mathrm{m}^{2}\right)$ & $30.24 \pm 5.18$ & $34.71 \pm 5.30$ & 0.001 \\
\hline Gestational weight gain (kg) & $16.05 \pm 5.43$ & $11.56 \pm 2.72$ & 0.001 \\
\hline Gestational weight gain $(\%)^{\mathrm{b}}$ & & & 0.011 \\
\hline Excessive & 44.1 & 61.2 & \\
\hline Adequate & 46.9 & 33.1 & \\
\hline Below & 9.0 & 5.6 & \\
\hline Glucose (mg/dl) & $72.39 \pm 7.12$ & $101.67 \pm 11.99$ & 0.001 \\
\hline İnsulin ( $\mu \mid \mathrm{U} / \mathrm{ml})$ & $8.07 \pm 2.02$ & $11.93 \pm 4.78$ & 0.001 \\
\hline HOMA-IR & $1.42 \pm 0.39$ & $3.06 \pm 1.26$ & 0.001 \\
\hline $\mathrm{HbA1c}(\%)$ & $5.01 \pm 0.32$ & $5.51 \pm 0.43$ & 0.001 \\
\hline Systolic BP (mmHg) & $108.06 \pm 8.74$ & $110.84 \pm 11.23$ & 0.052 \\
\hline Diastolic BP (mmHg) & $72.70 \pm 5.62$ & $73.48 \pm 5.11$ & 0.207 \\
\hline
\end{tabular}

Italics represents significant $\mathrm{p}$-values

PPO pre-pregnancy overweight/obesity, GDM gestational diabetes mellitus, GWG gestational weight gain, $B M I$ body mass index, $B P$ blood pressure, $H O M A-I R$ homeostasis model assessment-insulin resistance, $\mathrm{HbA1c}$ hemoglobin $\mathrm{A} 1 \mathrm{C}$

a Prepregnancy overweight/obesity is defined as the percentage of subjects with having $B M I \geq 25 \mathrm{~kg} / \mathrm{m}^{2}$

b Recommended gestational weight gain was calculated based on Institute of Medicine (IOM) recommendations according to pre-pregnancy BMI

Table 2 Minor allele frequency of polymorphisms

\begin{tabular}{lll}
\hline & Risk allele & $\begin{array}{l}\text { MAF } \\
\text { for study } \\
\text { sample }\end{array}$ \\
\hline HNF1A I27L rs1169288 & T & 0.44 \\
HNF1A S487N rs2464196 & T & 0.37 \\
HNF1A A98V rs1800574 & T & 0.10 \\
VDR Apal rs7975232 & $\mathrm{C}$ & 0.42 \\
VDR Taql rs731236 & $\mathrm{C}$ & 0.35 \\
VDR Bsml rs1544410 & $\mathrm{G}$ & 0.45 \\
VDR Fokl rs2228570 & $\mathrm{T}$ & 0.35 \\
FTO rs9939609 & $\mathrm{A}$ & 0.37 \\
\hline
\end{tabular}

MAF minor allele frequency

associated with excessive GWG risk compared with the $\mathrm{TT}$ and AT genotype $(\mathrm{OR}=1.73,95 \%$ CI [1.62-3.15], $\mathrm{p}=0.034$ ); however, this association was lost after adjusting for pre-pregnancy weight $(\mathrm{OR}=1.1,95 \% \mathrm{CI}$ [0.94-2.38], $\mathrm{p}>0.05$ ).

The HNF1A gene p.I27L distribution of GG-wild, GT, and TT was $34.5 \%, 53.8 \%$, and $11.7 \%$ in the controls, and $20.6 \%, 58.8 \%$, and $20.6 \%$ in women with GDM $(\mathrm{p}=0.009)$. The HNF1A gene p.I27L TT/GT genotype was associated with a greater risk of GDM in comparison with the GG genotype in co-dominant, dominant, and recessive models (dominant: $\mathrm{GT}+\mathrm{TT}$ vs. GG-wild, 79.4 vs. $65.5 \%$, OR $=2.02$, $95 \%$ CI $[1.21-$ 3.38], $\mathrm{p}=0.007$ and recessive: TT vs. $\mathrm{GT}+\mathrm{GG}, 20.6$ vs. $11.7 \%, \mathrm{OR}=1.95,95 \% \mathrm{CI}[1.13-3.49], \mathrm{p}=0.036$ ) (Table 3). Pre-pregnancy weight, weight at delivery, and GWG were similar between p.I27L genotypes $(\mathrm{p}>0.05)$ (Table 5). Glucose, HOMA-IR, and HbA1c were increased in the p.I27L TT genotype compared with the GG-wild type $(\mathrm{p}<0.05)$. Pre-pregnancy weight, weight at delivery, and GWG did not differ between the $V D R$ and HNF1A gene carriers ( $\mathrm{p}>0.05)$. 
Table 3 Genotype analysis of HNF1A gene, VDR gene and FTO gene polymorphisms

\begin{tabular}{|c|c|c|c|c|}
\hline & Controls, $\mathrm{n}$ & Gestational diabetes, $\mathrm{n}$ & OR $(95 \% \mathrm{Cl})$ & $p$ \\
\hline FTO gene rs9939609 (\%) & & & & $0.011^{*}$ \\
\hline Co-dominant wild type TT & 73 & 59 & & \\
\hline Heterozygous AT & 54 & 62 & $1.42(0.86-2.24)$ & $0.169^{* *}$ \\
\hline Homozygous AA & 18 & 39 & $2.68(1.39-4.13)$ & $0.003^{* * *}$ \\
\hline Dominant $(\mathrm{AT}+\mathrm{AA} / \mathrm{TT})$ & 72 vs. 73 & 101 vs. 59 & $1.73(1.12-2.74)$ & 0.018 \\
\hline Recessive (AA/AT + TT) & 18 vs. 127 & 39 vs. 121 & $2.27(1.23-4.19)$ & 0.007 \\
\hline HNF1 gene I27L rs1 169288 (\%) & & & & $0.009^{*}$ \\
\hline Co-dominant wild type GG & 50 & 33 & & \\
\hline Heterozygous GT & 78 & 94 & $1.82(1.13-3.12)$ & $0.026^{* *}$ \\
\hline Homozygous TT & 17 & 33 & $2.94(1.41-4.16)$ & $0.003^{* * *}$ \\
\hline Dominant (GT+TT/GG) & 95 vs. 50 & 127 vs. 33 & $2.02(1.21-3.38)$ & 0.007 \\
\hline Recessive (TT/GT + GG) & 17 vs. 128 & 33 vs. 127 & $1.95(1.13-3.49)$ & 0.036 \\
\hline HNF1 gene S487N rs2464196 (\%) & & & & $0.919^{*}$ \\
\hline Co-dominant wild type CC & 61 & 64 & & \\
\hline Heterozygous CT & 62 & 72 & $1.10(0.67-1.80)$ & $0.684^{* *}$ \\
\hline Homozygous TT & 22 & 24 & $1.04(0.52-2.04)$ & $0.910^{* * *}$ \\
\hline Dominant (CT+TT/CC) & 84 vs. 61 & 96 vs. 64 & $1.11(0.70-1.76)$ & 0.683 \\
\hline Recessive $(\mathrm{TT} / \mathrm{CT}+\mathrm{CC})$ & 22 vs. 123 & 24 vs 136 & $0.98(0.52-1.84)$ & 0.966 \\
\hline HNF1 gene A98V rs1800574 (\%) & & & & $0.433^{*}$ \\
\hline Co-dominant wild type CC & 121 & 130 & & \\
\hline Heterozygous CT & 22 & 24 & $1.01(0.54-1.90)$ & $0.962^{* *}$ \\
\hline Homozygous TT & 2 & 6 & $2.79(0.55-12.45)$ & $0.196^{* * *}$ \\
\hline Dominant model (CT+TT/CC) & 24 vs. 121 & 30 vs. 130 & $1.16(0.64-2.10)$ & 0.615 \\
\hline Recessive model (TT/CT+CC) & 2 vs. 143 & 6 vs. 154 & $2.78(0.55-12.5)$ & 0.196 \\
\hline VDR gene Apal rs7975232 (\%) & & & & $0.199^{*}$ \\
\hline Co-dominant wild type AA & 52 & 48 & & \\
\hline Heterozygous AC & 73 & 78 & $1.15(0.69-1.91)$ & $0.571^{* *}$ \\
\hline Homozygous CC & 20 & 34 & $1.84(0.93-3.62)$ & $0.076^{* * *}$ \\
\hline Dominant $(A C+C C / A A)$ & 93 vs. 52 & 112 vs. 48 & $1.30(0.80-2.10)$ & 0.279 \\
\hline Recessive $(C C / A A+A C)$ & 20 vs. 125 & 34 vs. 126 & $1.68(0.92-3.02)$ & 0.088 \\
\hline VDR gene Taql rs731236 (\%) & & & & $0.472^{*}$ \\
\hline Co-dominant wild type TT & 82 & 81 & & \\
\hline Heterozygous CT & 33 & 37 & $1.13(0.64-1.98)$ & $0.658^{* *}$ \\
\hline Homozygous CC & 30 & 42 & $1.41(0.80-2.48)$ & $0.222^{* * *}$ \\
\hline Dominant $(\mathrm{CT}+\mathrm{CC} / \mathrm{TT})$ & 63 vs. 82 & 79 vs. 81 & $1.26(0.82-2.04)$ & 0.301 \\
\hline Recessive $(\mathrm{CC} / \mathrm{CT}+\mathrm{TT})$ & 30 vs. 115 & 42 vs. 118 & $1.36(0.81-2.32)$ & 0.253 \\
\hline VDR gene Bsml rs 1544410 (\%) & & & & $0.461^{*}$ \\
\hline Co-dominant wild type AA & 57 & 53 & & \\
\hline Heterozygous AG & 52 & 63 & $1.32(0.78-2.24)$ & $0.290^{* *}$ \\
\hline Homozygous GG & 36 & 45 & $1.37(0.76-2.44)$ & $0.284^{* * *}$ \\
\hline Dominant $(A G+G G / A A)$ & 88 vs. 57 & 108 vs. 53 & $1.34(0.841-2.15)$ & 0.215 \\
\hline Recessive $(G G / A G+A A)$ & 36 vs. 109 & 45 vs. 116 & $1.18(0.71-1.97)$ & 0.515 \\
\hline VDR gene Fokl rs2228570 (\%) & & & & $0.191^{*}$ \\
\hline Co-dominant wild type CC & 78 & 76 & & \\
\hline Heterozygous CT & 43 & 44 & $1.05(0.62-1.77)$ & $0.855^{* *}$ \\
\hline Homozygous TT & 24 & 40 & $1.71(0.94-3.10)$ & $0.076^{* * *}$ \\
\hline
\end{tabular}


Table 3 (continued)

\begin{tabular}{|c|c|c|c|c|}
\hline & Controls, n & Gestational diabetes, n & OR $(95 \% \mathrm{Cl})$ & $\mathrm{p}$ \\
\hline Dominant $(C T+T T / C C)$ & 67 vs. 78 & 84 vs. 76 & $1.28(0.82-2.01)$ & 0.272 \\
\hline Recessive (TT/CT + CC) & 24 vs. 121 & 40 vs. 120 & $1.68(0.95-2.59)$ & 0.070 \\
\hline
\end{tabular}

Categorical variables were analyzed with Chi-square test or Fisher's exact test, where appropriate. Multiple logistic regression analysis and Fisher's exact test were tested using models: dominant (major allele homozygotes vs heterozygotes + minor allele homozygotes), recessive (major allele homozygotes + heterozygotes vs minor allele homozygotes) and codominant (major allele homozygotes vs heterozygote and minor allele homozygotes vs major allele homozygotes)

Italics represents significant $\mathrm{p}$-values

*p Wild vs homozygous vs heterozygous

**p heterozygous vs wild

***p homozygous vs wild type

Table 4 Clinics of pregnants according to the FTO gene rs9939609 SNP

\begin{tabular}{|c|c|c|c|c|c|c|}
\hline & TT-wild $(n=132)$ & AT $(n=116)$ & $A A(n=57)$ & $\mathbf{p}^{*}$ & $p^{* *}$ & $\mathbf{p}^{* * *}$ \\
\hline Controls (\%) & $55.3(n=73)$ & $46.6(n=54)$ & $31.6(n=18)$ & 0.169 & 0.003 & 0.060 \\
\hline Gestational diabetes mellitus (\%) & $44.7(n=59)$ & $53.4(n=62)$ & $68.4(n=39)$ & & & \\
\hline Pre-pregnancy BMI (\%) & & & & $<0.001$ & 0.001 & 0.011 \\
\hline Underweight $(<20$ kg/m²) & 18.2 & 10.3 & 7.0 & & & \\
\hline Normal weight $(20-24.9$ kg/m²) & 33.3 & 16.4 & 12.3 & & & \\
\hline Overweight (25-29.9 kg/m²) & 19.7 & 44.8 & 26.3 & & & \\
\hline Obesity $(\geq 30$ kg/m²) & 28.8 & 28.4 & 54.4 & & & \\
\hline Pre-pregnancy overweight/obesity (\%) & $48.5(n=64)$ & $73.3(n=85)$ & $80.7(n=46)$ & $<0.001$ & 0.001 & 0.284 \\
\hline Gestational weight gain (\%) & & & & 0.001 & $<0.001$ & 0.014 \\
\hline Below & 12.1 & 3.4 & 3.6 & & & \\
\hline Adequate & 51.5 & 37.9 & 16.1 & & & \\
\hline Excessive & 36.4 & 58.6 & 80.4 & & & \\
\hline Excessive GWG (\%) & $36.4(n=48)$ & $58.6(n=68)$ & $80.4(n=46)$ & 0.001 & $<0.001$ & 0.003 \\
\hline Pre-pregnancy weight (kg) & $65.79 \pm 13.80$ & $69.69 \pm 11.31$ & $76.78 \pm 14.81$ & 0.016 & $<0.001$ & 0.001 \\
\hline Pre-pregnancy BMI (kg/m²) & $25.80 \pm 5.80$ & $27.46 \pm 5.03$ & $30.36 \pm 6.27$ & 0.017 & $<0.001$ & 0.001 \\
\hline Weight at delivery (kg) & $78.52 \pm 13.01$ & $83.84 \pm 9.80$ & $90.78 \pm 14.79$ & 0.001 & $<0.001$ & $<0.001$ \\
\hline BMI at delivery $\left(\mathrm{kg} / \mathrm{m}^{2}\right)$ & $30.77 \pm 5.56$ & $33.03 \pm 4.72$ & $35.86 \pm 6.22$ & 0.001 & $<0.001$ & 0.001 \\
\hline Gestational weight gain (kg) & $10.93 \pm 3.77$ & $12.93 \pm 2.31$ & $13.98 \pm 4.91$ & 0.029 & 0.021 & 0.654 \\
\hline Glucose (mg/dl) & $84.64 \pm 18.01$ & $88.06 \pm 17.65$ & $91.64 \pm 17.25$ & 0.134 & 0.014 & 0.207 \\
\hline İnsulin $(\mu \mathrm{lU} / \mathrm{ml})$ & $9.61 \pm 4.35$ & $10.18 \pm 3.72$ & $11.27 \pm 4.89$ & 0.315 & 0.039 & 0.148 \\
\hline HOMA-IR & $2.16 \pm 1.26$ & $2.33 \pm 1.18$ & $2.65 \pm 1.37$ & 0.307 & 0.033 & 0.159 \\
\hline HbA1c (\%) & $5.22 \pm 0.48$ & $5.24 \pm 0.41$ & $5.41 \pm 0.51$ & 0.685 & 0.018 & 0.027 \\
\hline Systolic BP (mmHg) & $110.41 \pm 9.59$ & $108.87 \pm 10.61$ & $108.77 \pm 10.74$ & 0.232 & 0.298 & 0.951 \\
\hline Diastolic BP (mmHg) & $73.74 \pm 5.43$ & $72.81 \pm 5.03$ & $72.24 \pm 5.75$ & 0.169 & 0.090 & 0.503 \\
\hline
\end{tabular}

Italics represents significant $\mathrm{p}$-values

$P P O$ pre-pregnancy overweight/obesity, GDM gestational diabetes mellitus, GWG gestational weight gain, $B M I$ body mass index, $B P$ blood pressure, $H O M A-I R$ homeostasis model assessment-insulin resistance, $\mathrm{HbA1c}$ hemoglobin A1c

*p TT wild type vs heterozygote AT

**p TT wild type vs homozygote AA

***p heterozygote AT vs homozygote AA

a Prepregnancy overweight/obesity is defined as the percentage of subjects with having $\mathrm{BMI} \geq 25 \mathrm{~kg} / \mathrm{m}^{2}$

b Recommended gestational weight gain was calculated based on Institute of Medicine (IOM) recommendations according to pre-pregnancy BMI

\section{Discussion}

Both the FTO AA genotype and HNF1A p.I27L GT/ TT genotype were associated with an increased risk of having GDM in Turkish women. However, the $V D R$ gene (p.ApaI, p.TaqI, p.FokI, p.BsmI) and HNF1A gene
(p.A98V, p.S487N) were not associated with having GDM. Insulin resistance and impaired glucose metabolism was observed in both p.I27L TT and FTO AA genotype carriers. The FTO AA genotype was associated with an increased risk for pre-pregnancy overweight/obesity, 
Table 5 Clinics of pregnant women according to the HNF1A gene p.127L

\begin{tabular}{|c|c|c|c|c|c|c|}
\hline & GG wild $(n=83)$ & $G T(n=172)$ & $\operatorname{TT}(n=50)$ & $\mathbf{p}^{*}$ & $\mathbf{p}^{* *}$ & $\mathrm{p}^{* * *}$ \\
\hline Controls (\%) & $60.2(n=50)$ & $45.3(n=78)$ & $34.0(n=17)$ & 0.026 & 0.003 & 0.153 \\
\hline Gestational diabetes mellitus (\%) & $39.8(n=33)$ & $54.7(n=94)$ & $66.0(n=33)$ & & & \\
\hline Pre-pregnancy BMI (\%) & & & & 0.653 & 0.622 & 0.695 \\
\hline Underweight $\left(<20 \mathrm{~kg} / \mathrm{m}^{2}\right)$ & 15.7 & 13.4 & 8.0 & & & \\
\hline Normal weight (20-24.9 kg/m²) & 21.7 & 23.3 & 24.0 & & & \\
\hline Overweight $\left(25-29.9 \mathrm{~kg} / \mathrm{m}^{2}\right)$ & 33.7 & 27.9 & 34.0 & & & \\
\hline Obesity $\left(\geq 30 \mathrm{~kg} / \mathrm{m}^{2}\right)$ & 28.9 & 35.5 & 34.0 & & & \\
\hline Pre-pregnancy overweight/obesity (\%) & $62.7(n=52)$ & $63.4(n=109)$ & $68.0(n=34)$ & 0.911 & 0.532 & 0.547 \\
\hline Gestational weight gain (\%) & & & & 0.112 & 0.804 & 0.342 \\
\hline Below & 3.6 & 9.4 & 6.0 & & & \\
\hline Adequate & 45.8 & 35.1 & 46.0 & & & \\
\hline Excessive & 50.6 & 55.6 & 48.0 & & & \\
\hline Excessive GWG (\%) & $50.6(n=42)$ & $55.8(n=96)$ & $48.0(n=24)$ & 0.434 & 0.771 & 0.329 \\
\hline Pre-pregnancy weight (kg) & $67.93 \pm 13.64$ & $70.12 \pm 14.28$ & $68.94 \pm 11.42$ & 0.247 & 0.665 & 0.592 \\
\hline Pre-pregnancy BMI $\left(\mathrm{kg} / \mathrm{m}^{2}\right)$ & $26.78 \pm 5.74$ & $27.51 \pm 6.06$ & $27.35 \pm 5.24$ & 0.364 & 0.568 & 0.870 \\
\hline Weight at delivery (kg) & $81.84 \pm 13.34$ & $83.56 \pm 13.50$ & $81.98 \pm 10.73$ & 0.338 & 0.951 & 0.445 \\
\hline BMI at delivery $\left(\mathrm{kg} / \mathrm{m}^{2}\right)$ & $32.25 \pm 5.66$ & $32.77 \pm 5.88$ & $32.50 \pm 5.13$ & 0.503 & 0.795 & 0.772 \\
\hline Gestational weight gain (kg) & $14.02 \pm 4.60$ & $13.67 \pm 4.83$ & $13.24 \pm 4.98$ & 0.583 & 0.359 & 0.434 \\
\hline Glucose (mg/dl) & $83.89 \pm 17.10$ & $86.88 \pm 17.69$ & $94.06 \pm 18.23$ & 0.203 & 0.002 & 0.013 \\
\hline İnsulin $(\mu \mid \mathrm{U} / \mathrm{ml})$ & $9.52 \pm 3.16$ & $10.30 \pm 4.76$ & $10.64 \pm 4.09$ & 0.215 & 0.108 & 0.681 \\
\hline HOMA-IR & $2.10 \pm 1.01$ & $2.36 \pm 1.37$ & $2.54 \pm 1.23$ & 0.155 & 0.045 & 0.470 \\
\hline $\mathrm{HbA1c}(\%)$ & $5.15 \pm 0.40$ & $5.29 \pm 0.50$ & $5.32 \pm 0.41$ & 0.048 & 0.037 & 0.736 \\
\hline Systolic BP (mmHg) & $109.93 \pm 10.07$ & $109.27 \pm 10.26$ & $109.70 \pm 10.37$ & 0.626 & 0.896 & 0.797 \\
\hline Diastolic BP (mmHg) & $73.20 \pm 5.47$ & $73.11 \pm 5.26$ & $72.94 \pm 5.63$ & 0.901 & 0.790 & 0.838 \\
\hline
\end{tabular}

Italics represents significant $\mathrm{p}$-values

PPO pre-pregnancy overweight/obesity, GDM gestational diabetes mellitus, GWG gestational weight gain, $B M I$ body mass index, $B P$ blood pressure, $H O M A-I R$ homeostasis model assessment-insulin resistance, $\mathrm{HbA1c}$ hemoglobin A1c

*p wild GG vs heterozygote GT

**p wild GG vs homozygote TT

***p heterozygote GT vs homozygote TT

a Prepregnancy overweight/obesity is defined as the percentage of subjects with having $\mathrm{BMI} \geq 25 \mathrm{~kg} / \mathrm{m}^{2}$

b Recommended GWG was calculated based on Institute of Medicine (IOM) recommendations according to pre-pregnancy BMI

but not associated with excessive GWG after adjusting for pre-pregnancy weight. The association of the adiposity-related gene FTO with GDM might be mediated by the effect of FTO on pre-pregnancy obesity. The diabetes-related p.I27L gene was associated with GDM by increasing insulin resistance.

Our results demonstrated that the VDR gene p.ApaI, p.TaqI, p.BsmI, and p.FokI genotypes were not associated with having GDM in Turkish women. The VDR gene and HNF1A gene SNPs were not associated with pre-pregnancy weight, weight at delivery, and GWG during pregnancy. The associations of the $V D R$ gene and HNF1A gene with pre-pregnancy weight, weight at delivery, and GWG have not been investigated in previous studies. El-Beshbishy et al. reported that p.BsmI and p.FokI were not associated with GDM in Saudi women [22]. Incompatible to our results, p.FokI
[23], p.ApaI, and p.TaqI [22] were associated with an increased risk of GDM in Iranian women [24]. We found that the HNF1A gene p.A98V and p.S487N were not associated with GDM in Turkish women. Zurawek et al. reported that p.I27L, p.A98V, and p.S487N were not associated with GDM in Polish women [25]. No relationship was reported between p.A98V and GDM in Danish women [12]; however, insulin secretion was decreased in p.A98V carriers without GDM [26], which is compensated by increasing insulin sensitivity [27]. Our data show that the HNF1A gene p.I27L GT/ GG genotype was associated with an increased risk of GDM $(\mathrm{OR}=2.02,95 \%$ CI [1.21-3.38], $\mathrm{p}=0.007)$. Prepregnancy weight, weight at delivery, and GWG were not associated with p.I27L genotypes. Insulin resistance and impaired glucose metabolism was observed in p.I27L TT carriers. We suggest that the diabetes-related 
p.I27L gene was associated with the increased risk of GDM by impairing glucose metabolism and increasing insulin resistance. Similarly, p.I27L was associated with an increased GDM risk in Scandinavian women by the effect of p.I27L on pancreas beta cell function [28] and insulin resistance [29]. Decreased beta cell function/transcriptional activity, decreased glucose-stimulated insulin secretion, increased insulin resistance, and increased type 2 diabetes risk have been found in p.I27L + p.S487N carriers (if also including p.A98V) [27, 30, 31]. HNF1A controls beta cell function by regulating target genes such as glucose transporter 2, liver pyruvate kinase, collectrin, hepatocyte growth factor activator, and HNF4A. Decreased HNF1A activity causes decreased beta cell mass and expression of these target genes, which lead to impaired insulin secretion $[17,18]$. Beta-cell dysfunction is more prone to developing impaired glucose tolerance during pregnancy [28].

The FTO gene AA genotype was associated with an increased risk of having GDM $(\mathrm{OR}=2.27,95 \%$ CI $[1.23-$ 4.19 ], $\mathrm{p}=0.007)$. The FTO AA genotype had a greater risk for pre-pregnancy overweight/obesity $(\mathrm{OR}=1.43$, 95\% CI [1.25-3.4], $\mathrm{p}=0.035$ ). The FTO AA genotype was not associated with GWG after adjusting for pre-pregnancy weight $(\mathrm{OR}=1.1,95 \% \mathrm{CI}[0.94-2.38], \mathrm{p}>0.05)$. Insulin resistance and impaired glucose metabolism were observed in FTO AA genotype carriers. We suggest that the adiposity-related gene FTO was associated with increased risk of GDM by increasing pre-pregnancy obesity. Similarly, previous studies have shown that the FTO rs9939609 AA genotype was associated with higher prepregnancy weight $[10,13,32]$. Lawlor et al. reported that maternal fat or fetal fat adiposity-related variants were not associated with excessive GWG, but the FTO gene was associated with pre-pregnancy overweight [33]. The FTO gene has a role in the regulation of adiposity-related phenotypes through the effect of FTO on weight gain during younger ages [34] and continues throughout life [10]. FTO is expressed in the hypothalamic region, which regulates appetite [35], and this would contribute to energy intake and body fat mass [36]. Our data demonstrated that FTO gene AA genotype carriers were heavier before pregnancy, but AA carriers did not have significant weight gain during pregnancy. Chiou et al. reported that the FTO gene was associated with pre-pregnancy obesity and a tendency to gain less weight throughout pregnancy [5]. Consistent with our data, the FTO gene was not associated with greater GWG after adjusting for pre-pregnancy BMI in Caucasian and African-American populations [37]. The FTO gene was not associated with GWG according to the period of pregnancy in British [33] and Brazilian women [10]. Moreover, GWG comprises other factors such as the fetus, amniotic fluid, and placenta [10]. Pregnant women have biologic, behavioral, and hormonal changes throughout pregnancy [11]. Pre-pregnancy body weight shows maternal nutritional changes before conception, whereas GWG represents fetal-maternal physiologic conditions associated with genetic and nutrition factors [1]. This could modify the genetic contributions of the maternal FTO, HNF1A, and $V D R$ gene variants on pre-gestational weight and GWG, as well as GDM [13,33]; however it is not fully known which of these conditions is more associated with these disorders.

There are some limitations in our study that should be considered. We did not report the GWG according to gestational weeks. The small sample size resulted in a lower power for investigating a significant effect of any of the HNF1A, VDR, and FTO gene SNPs on weight changes during pregnancy. Also, we did not control our data for confounding variables such as nutrition, education, smoking and parity.

\section{Conclusion}

Both the FTO AA genotype and HNF1A p.I27L GT/ TT genotype were associated with increased GDM risk in Turkish pregnant women. However, the $V D R$ gene p.ApaI, p.TaqI, p.FokI, p.BsmI and the $H N F 1 A$ gene p.A98V, p.S487N genotypes were not associated with having GDM. The diabetes-related p.I27L gene was associated with GDM by increasing insulin resistance. The diabetes-related $H N F 1 A$ p.I27L gene was associated with insulin resistance, which might contribute to developing GDM. The FTO AA genotype was associated with prepregnancy overweight/obesity, but did not contribute to significant weight gain during pregnancy. The adiposity-related gene FTO was associated with GDM by the effect of FTO on pre-pregnancy obesity. The FTO gene was associated with pre-pregnancy obesity, which might contribute to developing GDM. Genetic factors involved in GDM, pre-pregnancy weight, and GWG should be identified for the prevention of adverse complications of GDM and obesity during pregnancy. Further studies with multiethnic and larger populations are needed to find genetic variants related to GDM, pre-pregnancy obesity, and GWG during pregnancy.

\section{Abbreviations}

GDM: gestational diabetes mellitus; GWG: gestational weight gain; BMI: body mass index; SNPs: single nucleotide polymorphisms; IOM: Institute of Medicine; HNF1A: hepatocyte nuclear factor 1a; FTO: the fat mass and obesity associated gene; VDR: vitamin D receptor; HOMA-IR: homeostasis model assessment-insulin resistance; $\mathrm{HbA1c}$ : hemoglobin A1c. 


\section{Acknowledgements \\ Not applicable.}

\section{Authors' contributions}

SB, contributions to conception and design, or acquisition of data, or analysis and interpretation of data, involved in drafting the manuscript, NE and MU, contributions to conception and design, or acquisition of data, or analysis and interpretation of data; MK, MC and MH contribute to acquisition of data, or analysis and interpretation of data; $\mathrm{EC}$, revising it critically for important intellectual content; and have given final approval of the version to be published. All authors read and approved the final manuscript.

\section{Funding}

No funding sources for research.

\section{Availability of data and materials}

All data are freely available for scientific purpose.

\section{Ethics approval and consent to participate}

This study was approved by Diskapi Yildirim Beyazit Teaching and Research Hospital Ethics Board (Number.24.04.2015-13/25). Written informed consent was obtained from all subjects.

\section{Consent to publish}

Not applicable.

\section{Competing interests}

The authors declare that they have no competing interests.

\section{Author details}

${ }^{1}$ Department of Endocrinology and Metabolism, Ankara Diskapi Yildirim Beyazit Teaching and Training Research Hospital, Ankara, Turkey. ${ }^{2}$ Department of Medical Biology, Baskent University, Ankara, Turkey. ${ }^{3}$ Department of Genetic Research, Ankara Diskapi Yildirim Beyazit Teaching and Training Research Hospital, Ankara, Turkey. ${ }^{4}$ Department of Obstetrics and Gynecology, Gulhane School of Medicine, Ankara, Turkey. ${ }^{5}$ Department of Internal Medicine, Afyonkarahisar Saglik Bilimleri University, Afyon, Turkey. ${ }^{6}$ Department of Endocrinology and Metabolism, Afyonkarahisar Saglik Bilimleri University, Afyon, Turkey.

Received: 30 December 2018 Accepted: 8 May 2019

Published online: 14 May 2019

\section{References}

1. Bianchi C, de Gennaro G, Romano M, Aragona M, Battini L, Del Prato S, Bertolotto A. Pre-pregnancy obesity, gestational diabetes or gestational weight gain: which is the strongest predictor of pregnancy outcomes? Diabetes Res Clin Pract. 2018;144:286-93.

2. Viecceli C, Remonti LR, Hirakata VN, Mastella LS, Gnielka V, Oppermann MLR, Silveiro SP, Reichelt AJ. Weight gain adequacy and pregnancy outcomes in gestational diabetes: a meta-analysis. Obes Rev. 2017; 18:567-80.

3. Li C, Liu Y, Zhang W. Joint and independent associations of gestational weight gain and pre-pregnancy body mass index with outcomes of pregnancy in Chinese women: a retrospective cohort study. PLoS ONE. 2015;10:e0136850.

4. Blackwell SC, Landon MB, Mele L, Reddy UM, Casey BM, Wapner RJ, Varner MW, Rouse DJ, Thorp JM, Sciscione A, Eunice Kennedy Shriver National Institute of Child Health and Human Development (NICHD) MaternalFetal Medicine Units (MFMU) Network, et al. Relationship between excessive gestational weight gain and neonatal adiposity in women with mild gestational diabetes mellitus. Obstet Gynecol. 2016;128:1325-32.

5. Chiou Y-L, Hung C-H, Liao H-Y. The impact of prepregnancy body mass index and gestational weight gain on perinatal outcomes for women with gestational diabetes mellitus. Worldviews Evid Based Nurs. 2018;15:313-22.

6. Egan AM, Dennedy MC, Al-Ramli W, Heerey A, Avalos G, Dunne F. ATLANTIC-DIP: excessive gestational weight gain and pregnancy outcomes in women with gestational or pregestational diabetes mellitus. J Clin Endocrinol Metab. 2014;99:212-9.

7. Dias S, Pheiffer C, Abrahams Y, Rheeder P, Adam S. Molecular biomarkers for gestational diabetes mellitus. Sci: Int J Mol; 2018. p. 19.

8. Kawai VK, Levinson RT, Adefurin A, Kurnik D, Collier SP, Conway D, Stein CM. A genetic risk score that includes common type 2 diabetes risk variants is associated with gestational diabetes. Clin Endocrinol. 2017;87:149-55.

9. Cormier H, Vigneault J, Garneau V, Tchernof A, Vohl M-C, Weisnagel SJ, Robitaille J. An explained variance-based genetic risk score associated with gestational diabetes antecedent and with progression to prediabetes and type 2 diabetes: a cohort study. BJOG Int J Obstet Gynaecol. 2015:122:411-9.

10. Martins MC, Trujillo J, Farias DR, Struchiner CJ, Kac G. Association of the FTO (rs9939609) and MC4R (rs17782313) gene polymorphisms with maternal body weight during pregnancy. Nutrition. 2016;32:1223-30.

11. Warrington NM, Richmond R, Fenstra B, Myhre R, Gaillard R, Paternoster L, Wang CA, Beaumont RN, Das S, Murcia M, et al. Maternal and fetal genetic contribution to gestational weight gain. Int J Obes. 2018:2005(42):775-84.

12. Lauenborg J, Damm P, Ek J, Glümer C, Jørgensen T, Borch-Johnsen K, Vestergaard H, Hornnes P, Pedersen O, Hansen T. Studies of the Ala/Nal98 polymorphism of the hepatocyte nuclear factor-1alpha gene and the relationship to beta-cell function during an OGTT in glucose-tolerant women with and without previous gestational diabetes mellitus. Diabet Med J Br Diabet Assoc. 2004;21:1310-5.

13. Gaillard R, Durmuş B, Hofman A, Mackenbach JP, Steegers EAP, Jaddoe VWV. Risk factors and outcomes of maternal obesity and excessive weight gain during pregnancy. Obesity. 2013;21:1046-55.

14. Bid HK, Konwar R, Aggarwal CG, Gautam S, Saxena M, Nayak VL, Banerjee $M$. Vitamin D receptor (Fokl, Bsml and Taql) gene polymorphisms and type 2 diabetes mellitus: a North Indian study. Indian J Med Sci. 2009;63:187-94.

15. Zhang J, Li W, Liu J, Wu W, Ouyang H, Zhang Q, Wang Y, Liu L, Yang R, Liu $X$, et al. Polymorphisms in the vitamin $D$ receptor gene and type 1 diabetes mellitus risk: an update by meta-analysis. Mol Cell Endocrinol. 2012;355:135-42.

16. Xia Z, Hu Y, Zhang H, Han Z, Bai J, Fu S, Deng X, He Y. Association of vitamin $\mathrm{D}$ receptor Fok I and Bsm I polymorphisms with dyslipidemias in elderly male patients with type 2 diabetes. Nan Fang Yi Ke Da Xue Xue Bao. 2014;34:1562-8.

17. Balamurugan K, Bjørkhaug L, Mahajan S, Kanthimathi S, Njølstad PR, Srinivasan N, Mohan V, Radha V. Structure-function studies of HNF1A (MODY3) gene mutations in South Indian patients with monogenic diabetes. Clin Genet. 2016;90:186-495.

18. Yamagata K. Roles of HNF1 $a$ and HNF4a in pancreatic $\beta$-cells: lessons from a monogenic form of diabetes (MODY). Vitam Horm. 2014;95:407-23.

19. Kwak SH, Kim S-H, Cho YM, Go MJ, Cho YS, Choi SH, Moon MK, Jung HS, Shin HD, Kang HM, et al. A genome-wide association study of gestational diabetes mellitus in Korean women. Diabetes. 2012;61:531-41.

20. Klemetti M, Hiltunen LM, Heino S, Heinonen S, Kajantie E, Laivuori H. An obesity-related FTO variant and the risk of preeclampsia in a Finnish study population. J Pregnancy. 2011;2011:251470.

21. Institute of Medicine (US), National Research Council (US) Committee to Reexamine IOM Pregnancy Weight Guidelines. Weight gain during pregnancy: reexamining the guidelines. Washington, DC: National Academies Press; 2009 .

22. El-Beshbishy HA, Tawfeek MA, Taha IM, FadulElahi T, Shaheen AY, Bardi FA, Sultan II. Association of vitamin D receptor gene Bsml (A>G) and Fokl (C>T) polymorphism in gestational diabetes among Saudi Women. Pak J Med Sci. 2015;31:1328-33.

23. Aslani S, Hossein-Nezhad A, Mirzaei K, Maghbooli Z, Afshar AN, Karimi F. VDR Fokl polymorphism and its potential role in the pathogenesis of gestational diabetes mellitus and its complications. Gynecol Endocrinol. 2011;27:1055-60.

24. Rahmannezhad G, Mashayekhi FJ, Goodarzi MT, Rezvanfar MR, Sadeghi A. Association between vitamin $D$ receptor Apal and Taql gene polymorphisms and gestational diabetes mellitus in an Iranian pregnant women population. Gene. 2016;581:43-7. 
25. Zurawek M, Wender-Ozegowska E, Januszkiewicz-Lewandowska D, Zawiejska A, Nowak J. GCK and HNF1alpha mutations and polymorphisms in Polish women with gestational diabetes. Diabetes Res Clin Pract. 2007;76:157-8.

26. Urhammer SA, Fridberg M, Hansen T, Rasmussen SK, Møller AM, Clausen JO, Pedersen O. A prevalent amino acid polymorphism at codon 98 in the hepatocyte nuclear factor-1alpha gene is associated with reduced serum C-peptide and insulin responses to an oral glucose challenge. Diabetes. 1997:46:912-6.

27. Bergman BC, Howard D, Schauer IE, Maahs DM, Snell-Bergeon JK, Eckel $\mathrm{RH}$, Perreault $\mathrm{L}$, Rewers $\mathrm{M}$. Features of hepatic and skeletal muscle insulin resistance unique to type 1 diabetes. J Clin Endocrinol Metab. 2012;97:1663-72.

28. Shaat N, Karlsson E, Lernmark A, Ivarsson S, Lynch K, Parikh H, Almgren P, Berntorp K, Groop L. Common variants in MODY genes increase the risk of gestational diabetes mellitus. Diabetologia. 2006;49:1545-51.

29. Chiu KC, Chuang L-M, Chu A, Yoon C, Wang M. Comparison of the impact of the $127 \mathrm{~L}$ polymorphism of the hepatocyte nuclear factor1alpha on estimated and measured beta cell indices. Eur J Endocrinol. 2003;148:641-7.

30. Awa WL, Thon A, Raile K, Grulich-Henn J, Meissner T, Schober E, Holl RW, DPV-Wiss Study Group. Genetic and clinical characteristics of patients with HNF1A gene variations from the German-Austrian DPV database. Eur J Endocrinol. 2011;164:513-20.

31. Winckler W, Burtt NP, Holmkvist J, Cervin C, de Bakker PIW, Sun M, Almgren P, Tuomi T, Gaudet D, Hudson TJ, et al. Association of common variation in the HNF1alpha gene region with risk of type 2 diabetes. Diabetes. 2005;54:2336-42.
32. Andraweera PH, Dekker GA, Leemaqz S, McCowan L, Roberts CT, SCOPE consortium. The obesity associated FTO gene variant and the risk of adverse pregnancy outcomes: evidence from the SCOPE study. Obesity. 2016;24:2600-7.

33. Lawlor DA, Fraser A, Macdonald-Wallis C, Nelson SM, Palmer TM, Davey Smith G, Tilling K. Maternal and offspring adiposity-related genetic variants and gestational weight gain. Am J Clin Nutr. 2011;94:149-55.

34. Hardy R, Wills AK, Wong A, Elks CE, Wareham NJ, Loos RJF, Kuh D, Ong KK. Life course variations in the associations between FTO and MC4R gene variants and body size. Hum Mol Genet. 2010;19:545-52.

35. Gerken T, Girard CA, Tung Y-CL, Webby CJ, Saudek V, Hewitson KS, Yeo GSH, McDonough MA, Cunliffe S, MCNeill LA, et al. The obesity-associated FTO gene encodes a 2-oxoglutarate-dependent nucleic acid demethylase. Science. 2007;318:1469-72.

36. Meyre D, Proulx K, Kawagoe-Takaki H, Vatin V, Gutiérrez-Aguilar R, Lyon D, Ma M, Choquet H, Horber F, Van Hul W, et al. Prevalence of lossof-function FTO mutations in lean and obese individuals. Diabetes. 2010;59:311-8

37. Stuebe AM, Lyon H, Herring AH, Ghosh J, Wise A, North KE, Siega-Riz AM. Obesity and diabetes genetic variants associated with gestational weight gain. Am J Obstet Gynecol. 2010;203:283.e1-e17.

\section{Publisher's Note}

Springer Nature remains neutral with regard to jurisdictional claims in published maps and institutional affiliations.
Ready to submit your research? Choose BMC and benefit from:

- fast, convenient online submission

- thorough peer review by experienced researchers in your field

- rapid publication on acceptance

- support for research data, including large and complex data types

- gold Open Access which fosters wider collaboration and increased citations

- maximum visibility for your research: over 100M website views per year

At BMC, research is always in progress.

Learn more biomedcentral.com/submissions 